\title{
EQUIVALENCE OF PROJECTIONS
}

\author{
S. K. BERBERIAN
}

\begin{abstract}
THEOREM: An $A W^{*}$-algebra is the ring generated by its projections if and only if it has no abelian summand. COROLLARY: Every equivalence in an $4 W^{*}$-algebra may be implemented by $a$ partial isometry in the ring generated by the projections of the algebra. The corollary is extended to certain finite Baer *-rings.
\end{abstract}

An early proposition in the theory of von Neumann algebras is that every such algebra is the closed linear span of its projections. Dixmier observed that in a factorial algebra, the passage to the closure can be dispensed with if ring multiplication is also allowed: Every factorial von Neumann algebra is the algebra (sums, products and scalar multiples are the allowable operations) generated by its projections [2, Proposition 7]. Fillmore and Topping removed the restriction on the center as much as it can be removed: A von Neumann algebra is the algebra generated by its projections if and only if its abelian summand (if it has any) is finite-dimensional [8]; inspection of their arguments shows that the resuit is valid for $A W^{*}$-algebras.

What about the ring (differences and products the allowable operations) generated by the projections? The answer is favorable, but abelian summands must be banished altogether:

THEOREM 1. An $A W^{*}$-algebra is the ring generated by its projections if and only if it has no abelian summand.

COROLI.ARY. Every equivalence in an $A W^{*}$-aigebra may be implemented by a partia!' isometry in the ring generated by the projections of the algebra.

The following remarks review the definitions and motivate the results. It is instructive to eniarge the setting somewhat; in the following remarks, $A$ is a Baer *-ring [11], that is, an involutive ring such that the right annihilator of each subset is the principal right ideal generated by a projection (=selfadjoint idempotent).

1. Projections $e, f$ in $A$ are said to be equivalent (relative to $A$ ), written $e \sim f$, if there exists $w \in A$ such that $w^{*} w=e$ and $w^{*} w^{*}=f$; such an element

Received by the editors Julv 8, 197 :

AMS 1969 subject classifications. Primary 4665.

Key words and phrases. von Neumann algebra, $A W^{*}$-algebra, Baer *-ring.

(c) American Mathematical Society 1972 
$w$ is called a partial isometry, and is said to implement the equivalence $e \sim f$.

2. We write $A^{\circ}$ for the ring generated by the projections of $A$, and call it the reduced ring of $A$; thus $A^{\circ}$ is the set of all finite sums of elements of the form $\pm e_{1} e_{2} \cdots e_{n}$, where the $e_{i}$ are projections in $A$. Since $A^{\circ}$ is a *-subring of $A$ containing every projection of $A$, it is immediate that $A^{\circ}$ is also a Baer *-ring. If $e, f$ are projections in $A$, we write $e \sim^{\circ} f$ if $e, f$ are equivalent relative to $A^{\circ}$. The assertion of the corollary is that if $A$ is an $A W^{*}$-algebra, then $e \sim f$ iff $e \sim{ }^{\circ} f$.

3. A symmetry in $A$ is a selfadjoint unitary element $s \quad\left(s^{*}=s=s^{-1}\right)$. If $g$ is a projection, then $2 g-1$ is a symmetry and belongs to $A^{\circ}$. Conversely, if 2 is invertible in $A$ then every symmetry $s$ has this form, the projection $g$ being defined by $g=(1 / 2)(1+s)$; in this paper, we need (and henceforth do) consider only symmetries of this form. Projections $e, f$ are said to be exchanged by a symmetry if there exists a symmetry $s$ such that ses $=f$ (hence se is a partial isometry in $A^{\circ}$ that implements $\left.e \sim{ }^{\circ} f\right)$.

4. If $e \sim f$ and $e, f$ are orthogonal $(e f=0)$, then $e, f$ may be exchanged by a symmetry $s$, for example, $s=w^{*}+w^{*}+1-(e+f)$, where $w$ is any partial isometry implementing $e \sim f$ (one notes that $2(e+f)$ is invertible in $(e+f) A(e+f)\left[11\right.$, p. 39, Exercise 11]). Thus $e \sim f$ implies $e \sim{ }^{\circ} f$ when $e, f$ are orthogonal.

5. A projection $e$ in $A$ is said to be finite (relative to $A$ ) if the relations $e \sim f \leqq e$ imply $f=e$. An equivalent condition on $e$ is that there does not exist an infinite sequence of orthogonal, nonzero subprojections $e_{n}$ of $e$ such that $e_{1} \sim e_{2} \sim e_{3} \sim \cdots[11$, Theorems 37 and 38]; thus it is clear from Remark 4 that $e$ is finite relative to $A$ iff it is finite relative to $A^{\circ}$.

6. Under mild hypotheses on $A$, the rings $A$ and $A^{\circ}$ have the same central projections (that is, a projection commutes with every element of $A$ iff it commutes with every projection of $A$ ). For example, it suffices to assume that $A$ satisfies Axiom E of [11]. (Axiom E: If $e, f$ are projections such that $e A f \neq 0$, then $e, f$ have equivalent, nonzero subprojections.)

7. A projection $e$ in $A$ is said to be abelian (relative to $A$ ) if every subprojection of $e$ has the form he with $h$ a central projection in $A$ ([9, Lemma 4.7], [4, Lemme 3.3], [11, pp. 10 and 36, Theorem 13]). If $e$ is abelian relative to $A$, then it is abelian relative to $A^{\circ}$; the converse is true if $A$ and $A^{\circ}$ have the same central projections (cf. Remark 6).

8. It follows from Remarks 4-7 that (under mild hypotheses on $A$ ), in the decomposition of a Baer *-ring according to type (finite and infinite; discrete and continuous; Types I, II, III ; and Type $\mathrm{I}_{\kappa}$, modulo the usual uniqueness question $[10$, p. 471]), the same central projections occur for $A^{\circ}$ as for $A$ (see [4], [9], [11, Theorem 12]). So to speak, $A$ and $A^{\circ}$ have 
the 'same' structure. For $A W^{*}$-algebras, the corollary drives the last nail into the coffin.

We approach the proof of Theorem 1 through two lemmas. The first asserts (generalizing siightly a result of Fillmore and Topping [8]) that matrix rings over $C^{*}$-algebras are projection-generated:

LEMMA 1. If $A=B_{n}$, where $n \geqq 2$ and $B$ is a $C^{*}$-algebra with unity, then $A^{\circ}=A$.

Proof. Here $B_{n}$ denotes the *-algebra of all $n \times n$ matrices over $B$ (with *-transpose as the involution). It is shown in [8, p. 333] that $A$ is the algebra generated by its projections, thus it suffices to observe that $A^{\circ}$ is an algebra.

More generally, if $A=B_{n}$, where $n \geqq 2$ and $B$ is any complex *-algebra with unity, then $A^{\circ}$ is a subalgebra of $A$. The point is that for every complex number $\lambda$, the 'scalar' matrix $\operatorname{diag}(\lambda 1, \cdots, \lambda 1)$ belongs to $A^{\circ}$. (The case $n=2$ is illustrative. It is no loss of generality to suppose $|\lambda| \leqq 1 / 2$. Then, for a suitable real number $\alpha, 0 \leqq \alpha \leqq 1$, the matrix

is a projection in $A$. Let

$$
e=\left(\begin{array}{cc}
\alpha 1 & \lambda 1 \\
\lambda * 1 & 1-\alpha 1
\end{array}\right)
$$

$$
s=\left(\begin{array}{ll}
0 & 1 \\
1 & 0
\end{array}\right), \quad f=\left(\begin{array}{ll}
1 & 0 \\
0 & 0
\end{array}\right)
$$

Since $s$ is a symmetry, the calculation

$$
f e s f+s(\text { fesf }) s=\left(\begin{array}{cc}
\lambda 1 & 0 \\
0 & \lambda 1
\end{array}\right)
$$

shows that $\left.\operatorname{diag}(\lambda .1, \lambda 1) \in A^{\circ}.\right)$

I.EMMA 2. If $A$ is a finite $A W^{*}$-algebra of Type I, having no abelian summand, then $A=A^{\circ}$.

Proof. It suffices to show that $A^{\circ}$ contains every element $x$ of $A$ that is selfadjoint or skew-adjoint. As shown in [8, p. 334], there exist orthogonal projections $e, f, g$ commuting with $x$, such that $e+f+g=1$, $e \sim f$ and $g \sim h \leqq f$ for a suitable projection $h$. Then

$$
x=(e+f) x(e+f)+g x g .
$$

The first term of $(*)$ belongs to $(e+f) A(e+f)$, which is *-isomorphic to $(e A e)_{2}$. Similarly $g x g$ belongs to $(g+h) A(g+h)$, which is *-isomorphic to $(g . A g)_{2}$. Applying Lemma 1 to each term of $(*)$, we see that $x \in A^{\circ}$.

PROOF OF THEOREM 1. The "only if" part is obvious, since it is clear from the functional representation that in an abelian algebra, the elements in the ring generated by the projections can have only integral spectrum. 
Conversely, suppose $A$ is an $A W^{*}$-algebra having no abelian summand. Write $A=B \ominus C \oplus D$, with $B$ properly infinite, $C$ finite and continuous, and $D$ finite of Type I (cf. [9], [11, Theorem 12]). By hypothesis, Lemma 2 is applicable to $D$. Since each of $B$ and $C$ may be written as a $2 \times 2$ matrix algebra (cf. [9, Lemmas 4.5 and 4.12], [11, Theorems 45 and 49]), application of Lemma 1 concludes the proof.

PROOF OF THE COROLlaRY. Write the algebra as the sum of an abelian algebra (in which equivalence collapses to equality) and an algebra without abelian summand (in which, by Theorem 1, the ring generated by the projections contains everything-in particular the partial isometries).

The status of the corollary for Baer $*$-rings is uncertain. We next prove some general results on equivalence in Baer *-rings that lead to a new proof of the corollary in the case of von Neumann algebras.

The next theorem sharpens and extends a generalized comparability theorem of Fillmore [6, Theorem 3]:

THEOREM 2. Let $A$ be a Baer *-ring satisfying the (EP)-axiom and the (SR)-axiom. If $e, f$ is any pair of projections in $A$, then there exist orthogonal decompositions $e=e_{1}+e_{2}, f=f_{1}+f_{2}$ such that $e_{1}, f_{1}$ are exchangeable by a symmetry and $e_{2}, f_{2}$ have orthogonal central covers.

Proof. (See [11] for the definitions.) Let $e^{\prime}, f^{\prime}$ be the left and right projections of $e f$, and set $e^{\prime \prime}=e-e^{\prime}, f^{\prime \prime}=f-f^{\prime}$. Thus $e=e^{\prime}+e^{\prime \prime}, f=f^{\prime}+f^{\prime \prime}$ with $e^{\prime \prime} f=e f^{\prime \prime}=0$ and $e^{\prime}, f^{\prime}$ exchangeable by a symmetry $s^{\prime}$ in

$$
\left(e^{\prime} \cup f^{\prime}\right) A\left(e^{\prime} \cup f^{\prime}\right) .
$$

\{The projections $e^{\prime}, f^{\prime}$ are in position $p^{\prime}$ [12], thus, in the case of von Neumann algebras, $s^{\prime}$ exists by a theorem of Dixmier [2]. The proof for $A W^{*}$-algebras is given in [1, Appendix II, Theorem II.12]; the proof is easily adapted to the Baer *-ring case using results in $[11, \S 13]$. $\}$ By generalized comparability, there exist orthogonal decompositions $e^{\prime \prime}=e_{0}+e_{2}$, $f^{\prime \prime}=f_{0}+f_{2}$ such that $e_{0} \sim f_{0}$ and $e_{2}, f_{2}$ have orthogonal central covers (cf. [3, Theorem 6], [9, Theorem 5.6], [11, Theorem 57]). Since $e_{0} f_{0}=0$, there exists a symmetry $s_{0}$ in $\left(e_{0}+f_{0}\right) A\left(e_{0}+f_{0}\right)$ exchanging $e_{0}, f_{0}$ (remark 4 above). From the orthogonality of $e^{\prime} \cup f^{\prime}$ and $e_{0}+f_{0}$, it follows that the element

$$
s=s^{\prime}+s_{0}+\left[1-\left(e^{\prime} \cup f^{\prime}+e_{0}+f_{0}\right)\right]
$$

is a symmetry in $A$ exchanging $e_{1}=e^{\prime}+e_{0}$ and $f_{1}=f^{\prime}+f_{0}$.

THEOREM 3. Let $A$ be a Baer *-ring satisfying the (EP)-axiom and the $(S R)$-axiom. If $e, f$ are finite projections in $A$, then the following conditions 
are equivalent: (a) e f; (b) $e, f$ can be exchanged by a symmetry in $A$; (c) $e \sim{ }^{\circ}$.

Proof. Dropping down to $(\epsilon \cup f) A(e \cup f)$, we can suppose that $A$ is finite (cf. [4, Lemma 1.5], [9, Theorem 6.2], [11, Theorem 56]). It is trivial that (c) implies (a).

(a) implies (b). Write $e=e_{1}+e_{2}, f=f_{1}+f_{2}$ as in Theorem 2. In particular, $e_{1} \sim f_{1}$, therefore $e_{2}=e-e_{1} \sim f-f_{1}=f_{2}$ by finiteness (cf. [3, Lemme 4.12], [9, Theorem 5.7], [11, p. 88, Exercise 2]); since equivalent projections iave the same central cover, we conclude that $e_{2}=f_{2}=0$. Thus $e=\epsilon_{1}, f=f_{1}$ are exchangeable by a symmetry.

(b) implies (c). This is noted in remark 3 above.

An alternate proof of the corollary of Theorem 1 for a von Neumann algebra (avoiding Lemmas 1-2) can be given as follows. The finite summand is covered by Theorem 3 . The properly infinite summand is covered by the following special case of Theorem 1 (proved in conversation with J. P. Williams):

Lemma. If $A$ is a properly infinite von Neumann algebra, then $A^{\circ}=A$.

ProOF. (i) If $u \in A$ is unitary then, by a theorem of Fillmore [7], $u$ is the product of (four) symmetries in $A$, thus $u \in A^{\circ}$.

(ii) If $r \in A$ is selfadjoint, let $n$ be an integer such that $\|(1 / 2 n) r\| \leqq 1$ and write $(1 / 2 n) r=(1 / 2)(u+v)$ with $u, v$ unitaries in $A$ [5, p. 4, Proposition 3]; then $r=n(u+v) \in A^{\circ}$ by (i).

(iii) If $b \in A$ is invertible, then $b \in A^{\circ}$ by (i), (ii) and polar decomposition.

(iv) If $a \in A$ is arbitrary, let $n$ be an integer not in the spectrum of $a$; then $a-n 1 \in A^{\circ}$ by (iii), thus $a=(a-n 1)+n 1 \in A^{\circ}$.

\section{REFERENCES}

1. S. K. Berberian, The regular ring of a finite $A W^{*}$-algebra, Ph.D. Thesis, University of Chicago, Chicago, Ill., 1955.

2. J. Dixmier, Position relative de deux variétés linéaires fermées dans un espace de Hilbert, Revue Sci. 86 (1948), 387-399. MR 10, 546.

3. - Les anneaux d'opérat'urs de classe finie, Ann. Sci. École Norm. Sup. (3) 66 (1949), 209-261. MR 11, 370.

4. - Sur la réduction des anneaux d'opérateurs, Ann. Sci. École Norm. Sup. (3) 68 (1951), 185-202. MR 13, 471.

5. — Les algèbres d'opérateurs dans l'espace hilbertien (Algèbres de von Neumann), 2ième éd., Gauthier-Villars, Paris, 1969.

6. P. A. Fillmore, Perspectivity in projection lattices, Proc. Amer. Math. Soc. 16 (1965), 383-387. MR 31 \#622.

7. - , On products of symmetries, Canad. J. Math. 18 (1966), 897-900. MR 33 \#4696. 
8. P. A. Fillmore and D. M. Topping, Operator algebras generated by projections, Duke Math. J. 34 (1967), 333-336. MR 35 \#751.

9. I. Kaplansky, Projections in Banach algebras, Ann. of Math. (2) 53 (1951), 235-249. MR 13, 48.

10. - Algebras of type I, Ann. of Math. (2) 56 (1952), 460-472. MR 14, 291. 11. - Rings of operators, Benjamin, New York, 1968. MR 39 \#6092.

12. Z. Takeda and T. Turumaru, On the property "Position p"', Math. Japon. 2 (1952), 195-197. MR 14, 990.

Department of Mathematics, University of Texas at Austin, Austin, Texas 78712 